\title{
CDKN2AIP Induces Cellular Senescence and Apoptosis of Testicular Seminoma Tumor by Interacting with CARM1 and elF4B
}

Yuanzhen Zhang ( $\nabla$ zhangyuanzhen@whu.edu.cn )

Zhongnan Hospital of Wuhan University

\section{Cao yuming}

Zhongnan Hospital of Wuhan University

Chen zhenlie

Zhongnan Hospital of Wuhan University

Qin zihan

Zhongnan Hospital of Wuhan University

Qian kaiyu

Zhongnan Hospital of Wuhan University

Liu tongzu

Zhongnan Hospital of Wuhan University

Primary research

Keywords: CDKN2AIP, CARM1, elF4B, seminoma, senescence, apoptosis

Posted Date: September 9th, 2021

DOI: https://doi.org/10.21203/rs.3.rs-853234/v1

License: () (1) This work is licensed under a Creative Commons Attribution 4.0 International License.

Read Full License 


\section{Abstract}

\section{Background}

Testicular seminoma are relatively rare tumors, which are mostly detected in male population aged between 15 to 44 years old. Although previous researches identified several molecular biomarkers associated with testicular seminoma pathogenesis, the exact mechanism for testicular seminoma progression remains largely unknown. CDKN2AIP has been previously indicated as tumor suppressor in multiple malignant diseases, in this study we aimed to further discover its role in testicular seminoma and underlying molecular mechanisms

\section{Methods}

Retrospectively collected testicular seminoma clinical samples and normal tissues combined with NTERA-2 cell line models, as well as mice xenograft models were utilized for this study. CDKN2AIP expression and its interaction with CARM1 and elF4B were detected by qRT-PCR, western blot, immunofluorescence assay combined with Co-IP and IP-MS experiments. Cellular senescence and apoptosis were subsequently evaluated via SA-beta-gal staining assay and H3K9me3 activity experiments. Mice xenograft animal model was used for in-vivo validation study

\section{Results}

CDKN2AIP was highly-expressed in normal testis samples, and was significantly suppressed in testicular seminoma clinical samples and cell line models. Up-regulation of CDKN2AIP was significantly associated with inhibited testicular seminoma tumor growth and increased cellular senescence and apoptosis. Further experiments demonstrated CDKN2AIP exhibited anti-tumor activity via interaction with CARM and elF4

\section{Conclusion}

CDKN2AIP induced testicular seminoma cellular senescence by suppressing CARM1 expression and elF4B phosphorylation. CDKN2AIP-CARM1 and CDKN2AIP-elF4B interaction-induced tumor cell senescence and apoptosis might be potential druggable molecular pathway in testicular seminoma tumor pathogenesis and progression

\section{Highlights}

Testicular seminoma are relatively rare tumors, although previous researches identified several molecular biomarkers associated with testicular seminoma pathogenesis, the exact mechanism for testicular seminoma progression remains largely unknown. In this study we demonstrated that CDKN2 AIP was highly-expressed in normal testis samples, and was significantly suppressed in testicular seminoma clinical samples and cell line models. Up-regulation of CDKN2AIP was significantly associated with inhibited testicular seminoma tumor growth and increased cellular senescence and apoptosis. Further 
experiments demonstrated CDKN2AIP exhibited anti-tumor activity via interaction with CARM and elF4B. This study enlightened potential druggable molecular pathway in future testicular seminoma tumor therapy development

\section{Introduction}

For global population, the occurrence of testicular germ cell tumors (TGCT) are quite rare, and testicular seminoma is a subtype of TGCT, with disease occurrence rate less than 1 case per 100,000 population. testicular seminomas are most commonly diagnosed in male population subgroup aged between 15 to 44 years old [1]. Currently, diagnostic strategies of testicular seminomas include ultrasonography, magnetic resonance (MRI) in combination of pathological examination and serum biological markers[2]. As for therapeutic interventions for testicular seminoma patient, current standard of care includes chemoor radiotherapy with surgical orchiectomy. It has been suggested that several factors are associated with prognosis for testicular seminoma patients including degree of lymphatic invasion, extra testicular invasion, as well as serum marker levels[3].

Up to date, researchers have identified several recurrent chromosomal anomalies associated with testicular seminoma pathogenesis and progression. Among them, chromosome 12p amplification is the most commonly detected variation in multiple testicular seminoma subtypes[4,5]. Detailed analysis suggested that amplification of multiple genes on 12p including CCND2, KRAS, TNFRSF1A, etc. played important role in progression of testicular seminomas[6-9].

CDKN2AIP (CDKN2A Interacting Protein), also known as CARF (the Collaborator of ARF), is a newly discovered marker involved in tumor pathogenesis. CDKN2AIP has been shown to interact with ARF and it activates key tumor suppressor gene p53 via ARF-dependent or independent pathways, causing inhibition of tumor growth and tumor cell senescence[10-13]. Interestingly, abnormally-high expression of CDKN2AIP unexpectedly demonstrates promotive effects on cancer expansion, and researchers discovered that CDKN2AIP promotes cellular malignant transformation via transcriptional repression of p21[14]. Moreover, recent study also indicated that CDKN2AIP amplification induced tumor invasion and metastasis via transcriptional activation of Wnt/ $\beta$-Catenin and subsequent EMT enhancement[15].

Our previous exploration indicated that CDKN2AIP expression was also detected in human testicles, and loss of CDKN2AIP resulted in decline of male fertility, the above phenomenon prompted us to believe CDKN2AIP might participate in the physiological process of germ cell proliferation or malignant transformation. Therefore, in this study, we aimed to further discover the role of CDKN2AIP in testicular seminoma pathogenesis and disease progression.

\section{Material And Methods}

\section{Patient recruitment and sample collection}


Seminoma samples were provided by Human Genetics Resource Preservation Center of Hubei Province (Department of Biological Repositories, Zhongnan Hospital of Wuhan University), China, a member of International Society for Biological and Environmental Repositories (ID:49623232). All patients have signed the informed consent. Our study followed the ethical rules in accordance with the Declaration of Helsinki and was approved by the ethical reviewing committee of Zhongnan hospital of wuhan university (NO. 2021009K).

\section{RNA extraction and qRT-PCR experiments}

RNAs from cell line samples and clinical samples were extracted using RNAiso Plus agent (TaKaRa, Dalian, China) following the standardized protocol. Reverse transcription polymerase chain reaction was performed to generate cDNAs. mRNA expression was detected by qRT-PCR under parameters setting as $95^{\circ} \mathrm{C}$ for $25 \mathrm{~s}, 55^{\circ} \mathrm{C}$ for $30 \mathrm{~s}, 72^{\circ} \mathrm{C}$ for $90 \mathrm{~s}$, with total cycles of 40 . Primers used in this study were listed in detail (Supplementary Table 1).

\section{Cell line culturing}

Testicular seminoma tumor cell line (NTERA-2) was acquired from American Type Culture Collection (ATCC, Manassas, VA, USA) on March 10, 2021. RPMI-1640 medium combined with 10\% fetal bovine serum (FBS, Hyclone, South Logan, UT, USA). $100 \mathrm{IU} / \mathrm{mL}$ penicillin with $100 \mu \mathrm{g} / \mathrm{mL}$ streptomycin (Invitrogen, Carlsbad, CA, USA) were used for culture, with environmental setting under $37^{\circ} \mathrm{C}$, with $5 \%$ $\mathrm{CO} 2$.

\section{Western Blot}

$2 \times 10^{6}$ cells per cell group were firstly washed twice with cold PBS, resuspended and then treated with icecold cell lysis buffer RIPA agent (Beyotime, Shanghai, Chian) to extract total protein from the samples. Then BCA protein assay kit was used for sample protein level quantification. Then, protein samples were firstly separated by SDS-PAGE and $10 \%$ separating gels and samples were subsequently electroblotted onto PVDF membranes (Immobilon-P, Millipore, Billerica, MA). After blocking in Tris buffer (50 mM Tris, $\mathrm{pH} 7.5$ ) containing $5 \%$ skim milk, the membranes were then incubated overnight at $4^{\circ} \mathrm{C}$ with primary antibodies. After rinsing with the Tris-Buffered Salin and Tween buffer solution (TBST, Sigma-Aldrich, St. Louis, MO, USA), they were then incubated with the secondary antibody for $2 \mathrm{~h}$. Chemiluminescence was used to expose the protein bands on the membrane. Antibodies used in this study were shown in Supplementary Table 2.

\section{Plasmids}

Target genes including CDKN2AIP, CARM1 (Coactivator-associated arginine methyltransferase 1) were cloned by PCR using cDNA as template, then inserted into pcDNA3.1 vector for subsequent transfection

Co-immunoprecipitation (Co-IP) 
NTERA-2 cells transfected with tagged CDKN2AIP/CARM1 were cultured in 10-cm dishes. Then cell samples were treated in immunoprecipitation buffer (containing $20 \mathrm{mM}$ Tris- $\mathrm{HCl}, \mathrm{pH} 7.4,150 \mathrm{mM} \mathrm{NaCl}, 1$ mM EDTA, pH 8.0, 1\% NP-40, 1× Protease and Phosphatase Inhibitor). Then $1 \mu$ l FLAG/HA antibody or IgG and $10 \mu \mathrm{l}$ of Protein A/G magnetic beads (pre-washed with lysis buffer) were added and incubated for $5 \mathrm{~h}$ under $4{ }^{\circ} \mathrm{C}$ condition. Afterwards, the beads were sequentially rinsed with wash buffer. Finally, proteins were eluted from the beads using $2 \times$ SDS loading buffer and subsequently treated at $95^{\circ} \mathrm{C}$ for $10 \mathrm{~min}$ prior to SDS-PAGE and immunoblotting with FLAG and HA antibodies.

\section{Immunofluorescence (IF)}

Cells samples were firstly washed with phosphate-buffered saline (PBS), and then paraformaldehyde was used for cell fixation for $15 \mathrm{~min}$. Afterwards, cells were permeabilized with $0.5 \% \mathrm{NP}-40$ for 20 min, and then cells were blocked with $5 \%$ bovine serum albumin for $30 \mathrm{~min}$. Subsequently, cells were incubated with primary antibody for $2 \mathrm{~h}$ at RT, and subsequently incubated with fluorescein conjugated secondary antibody for $1 \mathrm{~h}$. Finally, the DAPI and confocal microscopy were used to stain and image the slides, respectively.

\section{Flow cytometric apoptosis analysis}

Apoptosis of NTERA-2 cell groups was analyzed by flow cytometry utilizing Annexin V-fluorescein isothiocyanate (AV-FITC) apoptosis detection kit (640932, BioLegend, San Diego, CA, USA). NTERA-2 cell groups were respectively harvested and further processed according to the standardized procedure for flow cytometric experiment after the initial incubation.

\section{Cellular senescence assay}

SA-beta-gal staining assay was performed according to previous description[16]. Each group of cells were stained for 8 hours before evaluation by counting at least 300 cells.

\section{SCID mice xenograft model}

Pathogen-free 8-10 weeks old BALB/c SCID mice were used. For testicular seminoma tumor cell inoculation, cells were harvested by trypsination and viable cells $\left(5 \times 10^{6}\right)$ were suspended in $1 \mathrm{ml}$ of cell culture medium. An aliquot of $200 \mu \mathrm{L}$ of cell suspension was injected subcutaneously for each SCID mouse from every treatment group consisting of 9-11 SCID mice. The mice bearing testicular seminomas were sacrificed when the tumor had reached maximal growth (up to $20 \%$ of the body weight of the animal at the beginning of the experiment) or started to ulcerate. Primary tumors were removed, weighed and fixed in $10 \%$ PFA in PBS.

\section{Statistical analysis}

Statistical analysis was conducted by software package (SPSS 21.0 for Windows, IBM-SPSS Inc., Chicago, IL). Data were presented as the mean SD of three independent experiments. Statistical test of 
differences between numerical data was performed by standard t-test. Pearson test was conducted to compare gene correlation. $\mathrm{P}<0.05$ was considered to indicate statistical significance.

\section{Results}

CDKN2AIP were specifically detected in human testis and was suppressed in testicular seminoma tumor cells

Firstly, in order to understand the special role of CDKN2AIP in human testis, multiple human organ tissue samples including liver, lung, intestine, uterus, ovary and testis were utilized for CDKN2AIP protein expression detection via western-blot assay. Results indicated that the highest expression of CDKN2AIP was detected in human testis sample (Figure 1A). Moreover, through histological inspection via HE staining, we acquired typical samples of testicular seminoma tumor tissue and adjacent non-cancerous tissue (Figure 1D). Subsequent qRT-PCR and western blot analysis demonstrated that the mRNA and protein level of CDKN2AIP was significantly lower in tumor tissues (Figure 1B-1C). Consistently, further immunohistochemical (IHC) study on three pairs of matched testicular seminoma tumor and adjacent non-cancerous tissue samples also indicated that the expression level of CKDN2AIP was significantly lower in testicular seminoma tumor samples, compared with matched non-cancerous tissue samples (Figure 1F).

In addition, to elucidate the localization of CKDN2AIP, immunofluorescence assay (Figure 1E) and analysis were performed and results demonstrated that CKDN2AIP expression was identified in several kinds of cells including spermatogonium, spermatocyte, and sperm cells in human testis tissue.

\section{CDKN2AIP interacted with CARM1 to exhibited promotive effects on cellular senescence}

Secondly, to further explore the detailed mechanism of CDKN2AIP on testicular seminoma pathogenesis, we utilized IP-MS assay on NTERA-2 tumor cell line to detect CDKN2AIP-interacting proteins. As a result, several interacting-proteins were identified including CARM1, elF4B, SEC24C, CTTN, HBB, NCL, POLR2A protein (Figure 2A). Subsequent Co-IP study also confirmed the CDKN2AIP - CARM1 protein interaction (Figure 2B). Therefore, we then aimed to further discover the impact of CDKN2AIP-CARM1 and CDKN2AIP-elF4B interaction on malignant behavior of testicular seminoma tumor cells. As shown in Figure 2C-2D, after transfection of CDKN2AIP-specific siRNA, we found that the mRNA and protein expression of CARM1 was significantly elevated, while CDKN2AIP overexpression vector transfection notably suppressed CARM1 mRNA and protein expression. Moreover, immunofluorescence experiment on NTERA-2 cell groups also demonstrated consistent results (Figure 2E). Subsequently, we utilized senescence-associated beta-galactosidase (SA-beta-gal) activity assay and immunofluorescent experiment to detect histone $\mathrm{H} 3 \mathrm{~K} 9 \mathrm{me} 3$ level, which reflects the degree of senescent associated heterochromatin foci (SAHF) formation, so as to evaluate the impact of CDKN2AIP expression modulation on tumor cell senescence. As a result, we found that NTERA-2 cell group transfected with CDKN2AIP overexpression vector significantly increased tumor cell senescence, compared with control cell group, as shown in Figure 2F-2G. 
Additionally, we aimed to further explore the impact of CARM1 modulation on CDKN2AIP expression and its biological significance. As a result, CARM1 suppression significantly up-regulated CDKN2AIP mRNA and protein expression level, and vice versa (Figure 3A-3B). Subsequent SA-beta-gal activity assay and H3K9me3 immunofluorescence experiment also provided consistent results that CARM1 suppression significantly promoted NTERA-2 cell senescence (Figure 3C-3E).

To confirm the above findings, we utilized sublethal dose of Adriamycin (ADM) to induce cell senescence and explore the modulative influences of ADM treatment on CDKN2AIP and CARM1 expression. As shown in Figure 4A and Figure 4H, both SA-beta-gal and H3K9me3 immunofluorescence assay indicated that ADM treatment definitely promoted cellular senescence, and qRT-PCR \& Western blot analysis indicated ADM treated NTERA-2 cell exhibited increased CDKN2AIP and suppressed CARM1 mRNA and protein expression level (Figure 4B-4C). Immunofluorescence assay also confirmed enhanced CDKN2AIP expression in NTERA-2 cells induced by ADM treatment (Figure 4D and Supplementary Figure 3C). Following studies also confirmed that CDKN2AIP specific siRNA transfection reversed ADM-induced cellular senescence (Figure 4E). Consistently, immunofluorescence assay also confirmed significantly suppressed CARM1 level in ADM treated tumor cells (Figure 4F and Supplementary Figure 3D), and SAbeta-gal assay also indicated that by increasing CARM1 expression level via transfection of overexpression plasmids, ADM induced cellular senescence could be notably reversed (Figure 4G).

\section{CDKN2AIP inhibited anti-apoptotic pathway by binding with elF4B protein}

As previously IP-MS assay results indicated interaction of CDKN2AIP with elF4B, we then performed Co-IP experiment to confirm CDKN2AIP - elF4B protein interaction (Figure 5A). Further experiments were designed to discover the modulative effects of CDKN2AIP on elF4B expression and the impact of the CDKN2AIP - elF4B interaction on testicular seminoma tumor pathogenesis and progression. As shown in Figure 5B, western blot analysis indicated that for cell groups transfected with CDKN2AIP overexpression plasmids, phosphorylated type of elF4B protein was significantly suppressed while total elF4B protein level exhibited non-significant change. To further explore the impact of CDKN2AIP - elF4B interaction on tumor cell apoptosis, flow cytometric apoptosis assay was conducted and results indicated that tumor cell groups transfected with CDKN2AIP overexpression plasmids demonstrated significantly promoted cellular apoptosis (Figure 5C-5D), compared with control group. Subsequent qRT-PCR and western blot experiment also confirmed that for tumor cell group transfected with CDKN2AIP overexpression plasmids, the mRNA and protein expression of pro-apoptotic gene Bax and TP53 was significant elevated, while the expression of anti-apoptotic gene BCL-2 was significantly suppressed (Figure 5E-5F).

\section{CDKN2AIP inhibited tumor expansion in mice xenograft model study}

Finally, in order to consolidate our findings, we utilized mice xenograft model to validate the inhibitory effects of CDKN2AIP on testicular seminoma tumor expansion. Firstly, NTERA-2 cells with or without transfection of CDKN2AIP overexpression plasmids were respectively inoculated in SCID mice, tumor growth was then monitored and tumor tissue samples were subsequently gathered to detect CDKN2AIP protein level. As a result, tumor sample IHC study confirmed significantly suppressed CDKN2AIP 
expression in xenograft tumor tissue (Figure 6D-6E). Tumor expansion monitoring results again demonstrated significant tumor growth suppression in mice group inoculated with CDKN2AIP overexpressed tumor cells (Figure 6A). Subsequent tumor volume and weight inspection also confirmed the significant inhibitory effects of CDKN2AIP overexpression on xenograft tumor expansion (Figure 6B$6 C)$.

\section{Discussion}

In this study, we demonstrated for the first time that expression of CDKN2AIP was exclusively high in human testis tissue, and similar expression pattern was also observed in mice testis tissue (Supplementary Fig. 3A). Additionally, sequence homology analysis demonstrated quite conserved CDKN2AIP protein sequences among different species, including human, rat and mouse (Supplementary Fig. 3B), which indicated CDKN2AIP possessed relatively conservative and fundamental role in regulation of biological function in normal cells. In our study we also unveiled that the suppression of CDKN2AIP was characteristic in testicular seminoma tumor cells, and CDKN2AIP served as inhibitory role in testicular seminoma tumor progression. As shown in Fig. 7, CDKN2AIP functions as tumor suppressor via two diverse routes, on one hand CDKN2AIP induced cellular senescence by interacting with CARM1, on the other hand, CDKN2AIP also induced apoptosis by interacting elF4B and reduced elF4B phosphorylation.

Beyond the conventional p53 mediated molecular pathway of CDKN2AIP to induce cellular senescence, further detailed study demonstrated that CDKN2AIP exerted its anti-tumor effects via interaction with CARM1 and elF4B. CARM1, also known as PRMT4, belongs to protein arginine methyltransferase (PRMT) family [17]. CARM1 exerts its expression regulatory function via methylation residues on multiple transcription factors, RNA polymerase II and other gene expression modulators [18]. Previous researches also indicated CARM1 was associated with several crucial molecular processes, including RNA processing [19], transcriptional activation [20], tumor cellular apoptosis, cellular growth and progression [21-23]. It has been demonstrated that CARM1 upregulation was associated with pathogenesis of several types of human cancers, including breast cancer, prostate cancer and colorectal cancer, and CARM1 facilitated tumor cell initiation, progression and metastasis [21]. Our study provided novel evidences that CDKN2AIP exhibited cellular senescence-inducing function by suppressing CARM1, which further expanded CDKN2AIP regulatory molecular network on tumor cellular senescence. And CARM1 might serve as potential therapeutic target for future testicular seminoma treatment.

Moreover, in this study we also demonstrated for the first time that CDKN2AIP exhibited anti-apoptotic functions against tumor cell survival by suppressing the phosphorylation of elF4B. As an RNA binding protein, elF4B stimulates translation via interaction with elF4A[24], and its activity was modulated via Ser422 or Ser406 phosphorylation through MAPK and PI3K-mTOR pathways[25, 26]. However, whether CDKN2AIP-CARM1 interaction had cross-talk with elF4B remained unknown. Therefore, more detailed molecular experiments should be required to fully unveil the regulator network of CDKN2AIP in tumor pathogenesis. Besides, due to the nature of CDKN2AIP's dual functions both in germ cell formation and 
testicular seminoma pathogenesis, it stands as representative for a novel cluster of genes we named as 'Cancerous-Testis Gene Cluster', which might include more candidates in future researches and deserve more thorough investigations.

Notably, as our study was generally based on in-vitro tumor cell line models and retrospective clinical cohorts, the evidences drew from our research should be further consolidated in future studies with expanded scale. In-vivo animal models with CDKN2AIP gene knock-down combined with perspective multi-centered clinical studies are also warranted for future explorations.

\section{Conclusions}

In this study, through clinical samples combined with in-vitro tumor cell line and mice xenograft animal model experiments, we demonstrated for the first time that CDKN2AIP induced testicular seminoma cellular senescence by suppressing CARM1 expression and elF4B phosphorylation. CDKN2AIP-CARM1 and CDKN2AIP-elF4B interaction-induced tumor cell senescence and apoptosis might be potential druggable molecular pathway in testicular seminoma tumor pathogenesis and progression, which would enlighten novel therapy development in future testicular seminoma-related researches.

\section{Declarations}

\section{Ethics approval and consent to participate}

The study was approved by the Ethics Review Committee of zhongnan Hospital of Wuhan university (Wuhan, China).

\section{Consent for publication}

Not applicable.

\section{Availability of data and materials}

The datasets used and/or analyzed during the current study are available from the corresponding author on reasonable request.

\section{Competing interests}

The authors declare that they have no competing interests.

\section{Funding}

This study was supported by a research grant (No.81771543) from the National Natural Science Foundation of China.

\section{Authors' contributions}


CYM and ZYZ designed the study, CYM, QZH, CZL, and QKY performed the experiments and prepared the figures, CYM, LTZ and ZYZ contributed to drafting the manuscript. All authors read and approved the final manuscript.

\section{Acknowledgements}

None.

\section{References}

1. Ghazarian AA, Kelly SP, Altekruse SF, Rosenberg PS, McGlynn KA. Future of testicular germ cell tumor incidence in the United States: Forecast through 2026. Cancer. 2017,123:2320-8.

2. Batool A, Karimi N, Wu X-N, Chen S-R, Liu Y-X. Testicular germ cell tumor: a comprehensive review. Cell Mol Life Sci. Switzerland, 2019,76:1713-27.

3. Vogt AP, Chen Z, Osunkoya AO. Rete testis invasion by malignant germ cell tumor and/or intratubular germ cell neoplasia: what is the significance of this finding? Hum Pathol. United States, 2010,41:1339-44.

4. Atkin NB, Baker MC. Specific chromosome change, i(12p), in testicular tumours? Lancet (London, England). England, 1982. p. 1349.

5. Stock C, Strehl S, Fink FM, Bauer S, Lion T, Kreczy A, et al. Isochromosome 12p and maternal loss of 1 p36 in a pediatric testicular germ cell tumor. Cancer Genet Cytogenet. United States, 1996,91:95100.

6. Rodriguez S, Jafer O, Goker H, Summersgill BM, Zafarana G, Gillis AJM, et al. Expression profile of genes from $12 p$ in testicular germ cell tumors of adolescents and adults associated with $i(12 p)$ and amplification at 12p11.2-p12.1. Oncogene. England, 2003,22:1880-91.

7. Ezeh UI, Turek PJ, Reijo RA, Clark AT. Human embryonic stem cell genes OCT4, NANOG, STELLAR, and GDF3 are expressed in both seminoma and breast carcinoma. Cancer. United States, 2005,104:2255-65.

8. Korkola JE, Houldsworth J, Chadalavada RS V, Olshen AB, Dobrzynski D, Reuter VE, et al. Downregulation of stem cell genes, including those in a $200-\mathrm{kb}$ gene cluster at $12 \mathrm{p} 13.31$, is associated with in vivo differentiation of human male germ cell tumors. Cancer Res. United States, 2006,66:820-7.

9. Juric D, Sale S, Hromas RA, Yu R, Wang Y, Duran GE, et al. Gene expression profiling differentiates germ cell tumors from other cancers and defines subtype-specific signatures. Proc Natl Acad Sci U S A. 2005,102:17763-8.

10. Cheung CT, Singh R, Kalra RS, Kaul SC, Wadhwa R. Collaborator of ARF (CARF) regulates proliferative fate of human cells by dose-dependent regulation of DNA damage signaling. J Biol Chem. 2014,289:18258-69. 
11. Hasan K, Cheung C, Kaul Z, Shah N, Sakaushi S, Sugimoto K, et al. CARF Is a vital dual regulator of cellular senescence and apoptosis. J Biol Chem. United States, 2009,284:1664-72.

12. Hasan MK, Yaguchi T, Harada JI, Hirano T, Wadhwa R, Kaul SC. CARF (collaborator of ARF) interacts with HDM2: evidence for a novel regulatory feedback regulation of CARF-p53-HDM2-p21WAF1 pathway. Int J Oncol. Greece, 2008,32:663-71.

13. Hasan MK, Yaguchi T, Minoda $Y$, Hirano T, Taira $K$, Wadhwa R, et al. Alternative reading frame protein (ARF)-independent function of CARF (collaborator of ARF) involves its interactions with p53: evidence for a novel p53-activation pathway and its negative feedback control. Biochem J. 2004,380:605-10.

14. Kalra RS, Cheung CT, Chaudhary A, Prakash J, Kaul SC, Wadhwa R. CARF (Collaborator of ARF) overexpression in p53-deficient cells promotes carcinogenesis. Mol Oncol. 2015,9:1877-89.

15. Kalra RS, Chaudhary A, Yoon A-R, Bhargava P, Omar A, Garg S, et al. CARF enrichment promotes epithelial-mesenchymal transition via Wnt/ $\beta$-catenin signaling: its clinical relevance and potential as a therapeutic target. Oncogenesis. 2018,7:39.

16. Gewinner C, Wang ZC, Richardson A, Teruya-Feldstein J, Etemadmoghadam D, Bowtell D, et al. Evidence that inositol polyphosphate 4-phosphatase type II is a tumor suppressor that inhibits PI3K signaling. Cancer Cell. 2009,16:115-25.

17. Teyssier $C$, Chen $D$, Stallcup MR. Requirement for multiple domains of the protein arginine methyltransferase CARM1 in its transcriptional coactivator function. J Biol Chem. United States, 2002,277:46066-72.

18. Shishkova E, Zeng H, Liu F, Kwiecien NW, Hebert AS, Coon JJ, et al. Global mapping of CARM1 substrates defines enzyme specificity and substrate recognition. Nat Commun. 2017,8:15571.

19. Ohkura N, Takahashi M, Yaguchi $H$, Nagamura $Y$, Tsukada T. Coactivator-associated arginine methyltransferase 1, CARM1, affects pre-mRNA splicing in an isoform-specific manner. J Biol Chem. United States, 2005,280:28927-35.

20. Hu JL, Wang W, Lan XL, Zeng ZC, Liang YS, Yan YR, et al. CAFs secreted exosomes promote metastasis and chemotherapy resistance by enhancing cell stemness and epithelial-mesenchymal transition in colorectal cancer. Mol Cancer. 2019,18:91.

21. Yang Y, Bedford MT. Protein arginine methyltransferases and cancer. Nat Rev Cancer. England, 2013,13:37-50.

22. O'Brien KB, Alberich-Jordà M, Yadav N, Kocher O, Diruscio A, Ebralidze A, et al. CARM1 is required for proper control of proliferation and differentiation of pulmonary epithelial cells. Development. 2010,137:2147-56.

23. Kim D, Park M, Lim S, Choi J, Kim J, Han H, et al. High-glucose-induced CARM1 expression regulates apoptosis of human retinal pigment epithelial cells via histone 3 arginine 17 dimethylation: role in diabetic retinopathy. Arch Biochem Biophys. United States, 2014,560:36-43.

24. Rozen F, Edery I, Meerovitch K, Dever TE, Merrick WC, Sonenberg N. Bidirectional RNA helicase activity of eucaryotic translation initiation factors 4A and 4F. Mol Cell Biol. 1990,10:1134-44. 
25. Shahbazian D, Roux PP, Mieulet V, Cohen MS, Raught B, Taunton J, et al. The mTOR/PI3K and MAPK pathways converge on elF4B to control its phosphorylation and activity. EMBO J. 2006,25:2781-91.

26. Holz MK, Ballif BA, Gygi SP, Blenis J. mTOR and S6K1 mediate assembly of the translation preinitiation complex through dynamic protein interchange and ordered phosphorylation events. Cell. United States, 2005,123:569-80.

\section{Figures}


A

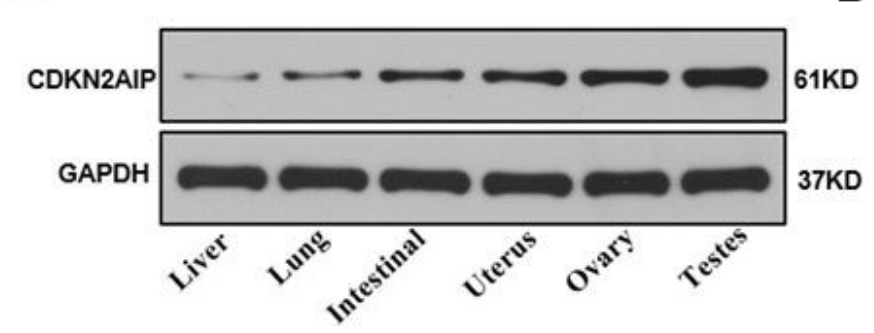

C
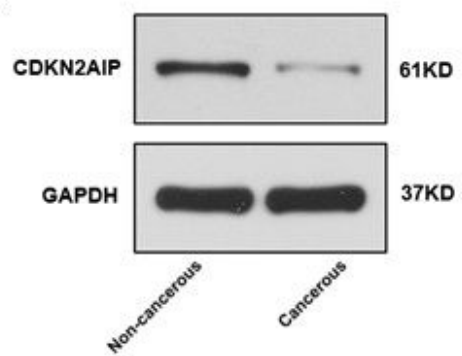

D

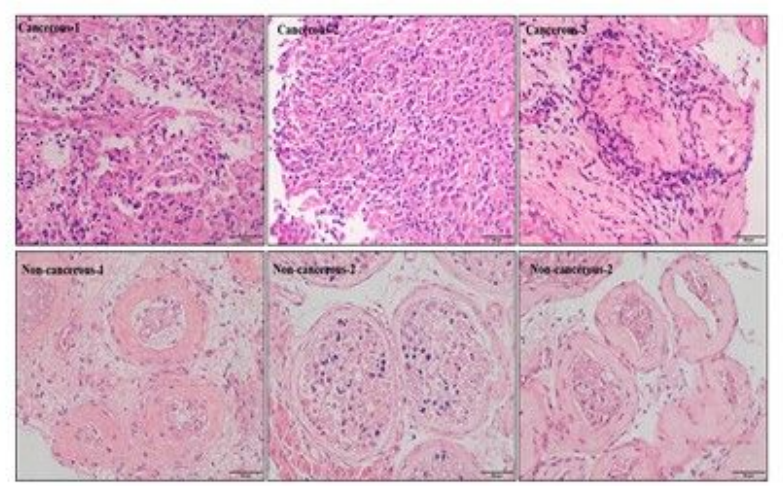

$\mathbf{E}$

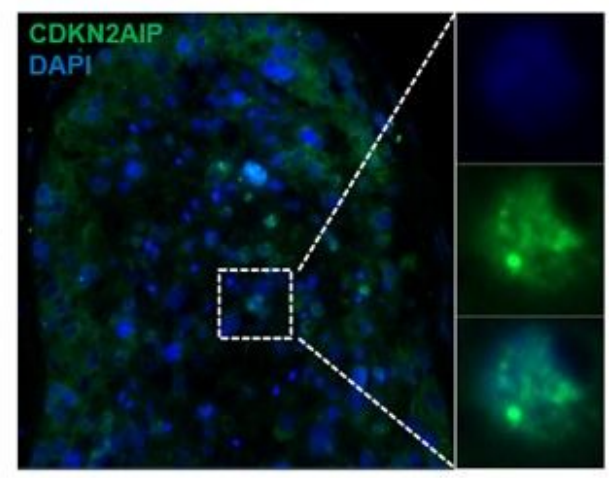

$\mathbf{F}$
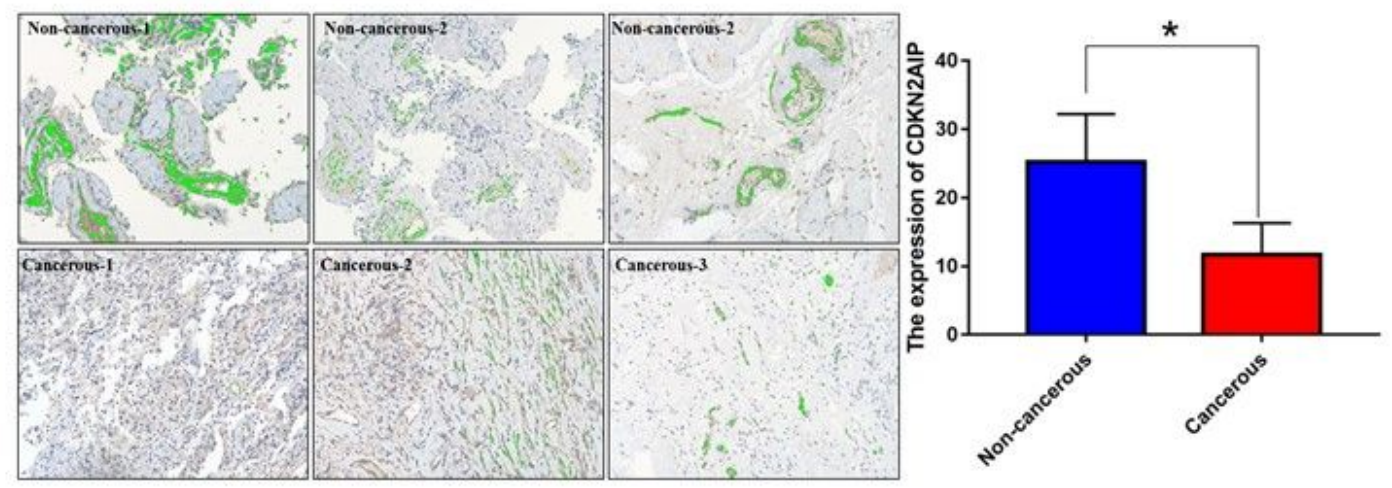

\section{Figure 1}

Detection of CDKN2AIP expression in normal tissues and testicular seminoma samples A. Western Blot detection of CDKN2AIP protein expression in several kinds of human normal tissues, including testes, liver, lung, small intestine, uterus and ovary B-C. Western Blot and qRT-PCR analysis on CDKN2AIP protein expression in testicular seminoma tumor and matched adjacent normal tissues D. HE-staining slides of testicular seminoma tumor tissue and matched adjacent normal tissue E. Immunofluorescence assay to 
detect the localization of CDKN2AIP protein in multiple kinds of cells including spermatogonium, spermatocyte, and sperm in human testis F. IHC assay to detect CKDN2AIP protein expression in three pairs of matched testicular seminoma tumor and adjacent normal tissues

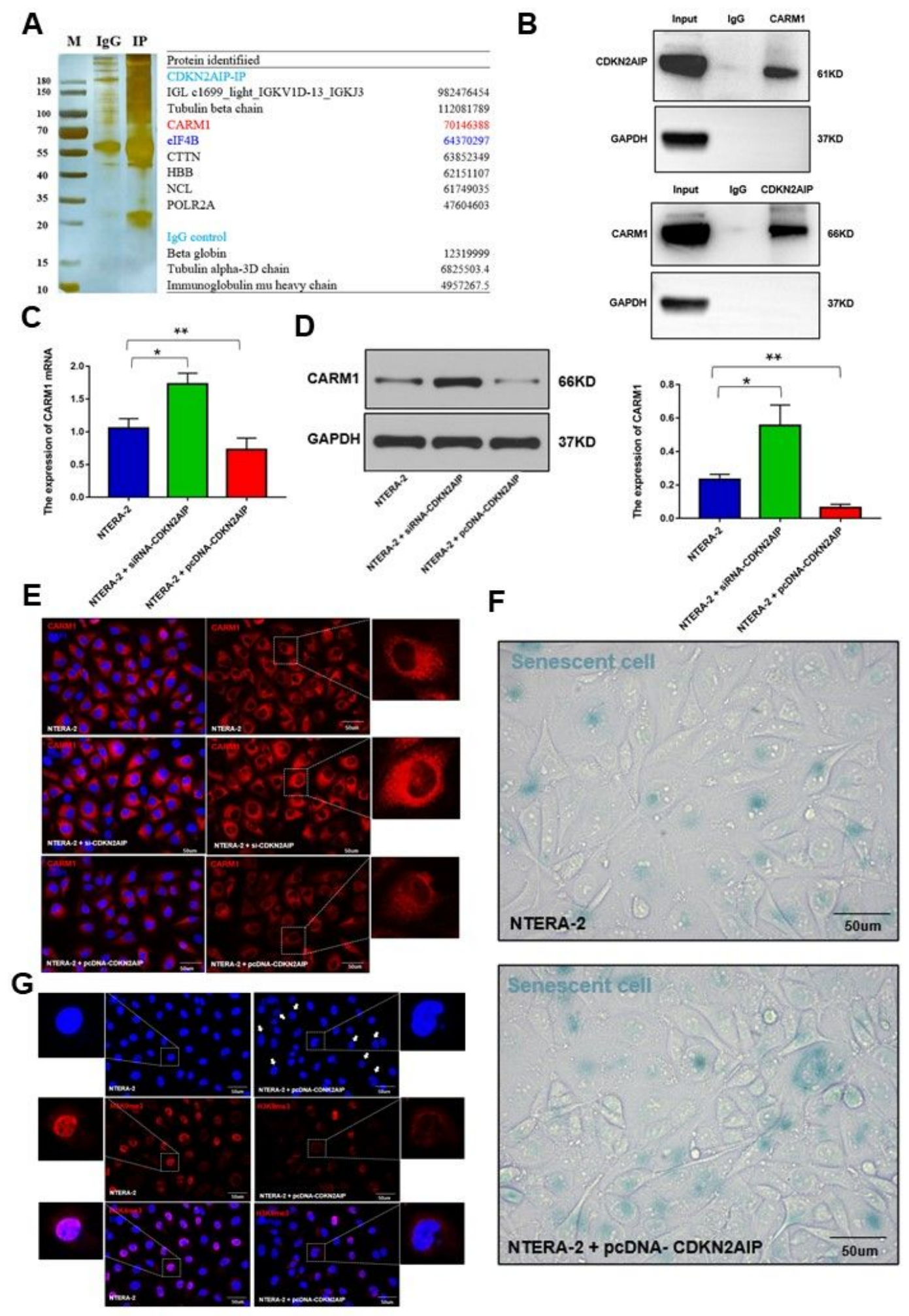

Figure 2

Detection of CDKN2AIP interaction with CARM1 in NTERA-2 tumor cell line and evaluation of CDKN2AIP modulation on cellular senescence A. IP-MS experiment to detect CDKN2AIP interacting proteins in 
NTERA-2 tumor cell line. Enrichment fold level of each interacting protein detected in CDKN2AIP-IP group or IgG control group was listed in detail in the right table B. Co-IP validation experiment to confirm the interaction of CDKN2AIP and CARM1 in NTERA-2 cell line C-D. qRT-PCR and Western-Blot analysis on CARM1 mRNA and protein expression level in NTERA-2 cell groups respectively transfected with CDKN2AIP-specific siRNA or overexpression vectors. E. Immunofluorescence detection of CARM1 in NTERA-2 cell groups respectively transfected with CDKN2AIP-specific siRNA or overexpression vectors. F. Senescence-associated beta-galactosidase (SA-beta-gal) activity assay on NTERA-2 cell groups respectively transfected with CDKN2AIP-specific siRNA or overexpression vectors. G. Immunofluorescence assay to detect histone $\mathrm{H} 3 \mathrm{~K} 9 \mathrm{me} 3$ level as senescent associated heterochromatin foci (SAHF) marker in NTERA-2 cell groups respectively transfected with CDKN2AIP-specific siRNA or overexpression vectors. 

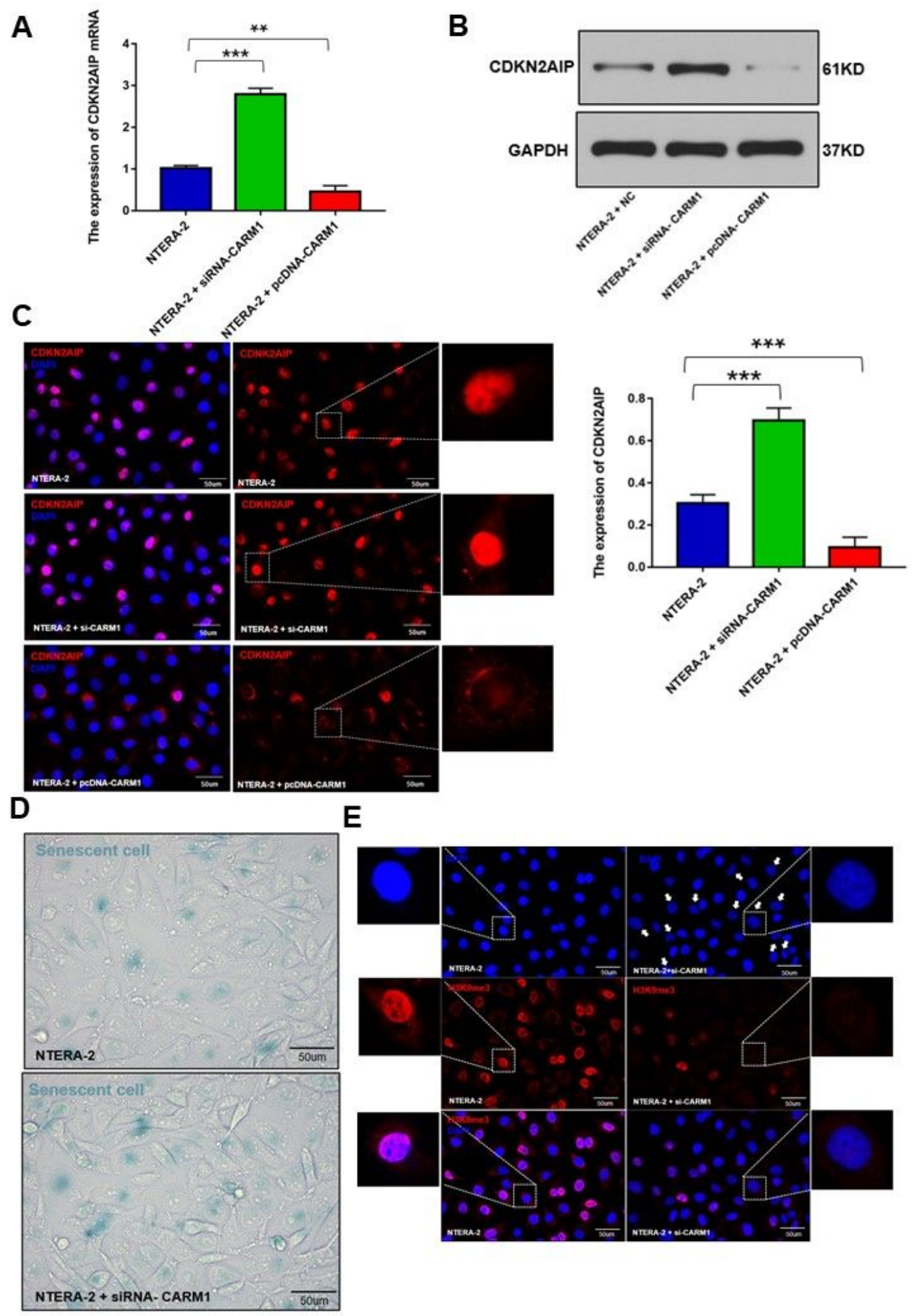

Figure 3

Evaluation of the impact of CARM1 modulation on CDKN2AIP expression and cellular senescence A. qRTPCR experiment to detect CDKN2AIP mRNA expression level in NTERA-2 cell groups respectively transfected with CARM1-specific siRNA or overexpression vectors B. Western Blot analysis on CDKN2AIP protein expression level in NTERA-2 cell groups respectively transfected with CARM1-specific siRNA or overexpression vectors C. Immunofluorescence detection of CDKN2AIP expression level in NTERA-2 cell 
groups respectively transfected with CARM1-specific siRNA or overexpression vectors D. Senescenceassociated beta-galactosidase (SA-beta-gal) activity assay on NTERA-2 cell groups respectively transfected with CARM1-specific siRNA or overexpression vectors. E. Immunofluorescence assay to detect histone H3K9me3 level in NTERA-2 cell groups respectively transfected with CARM1-specific siRNA or overexpression vectors.

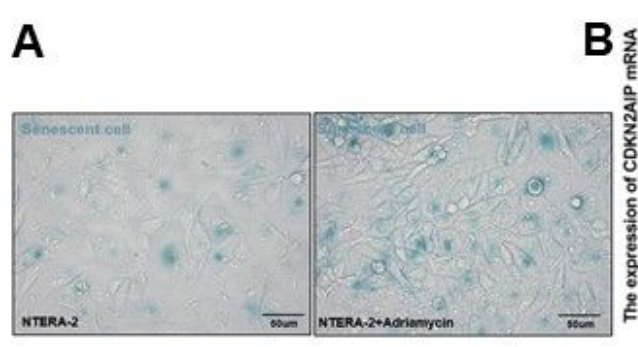

C
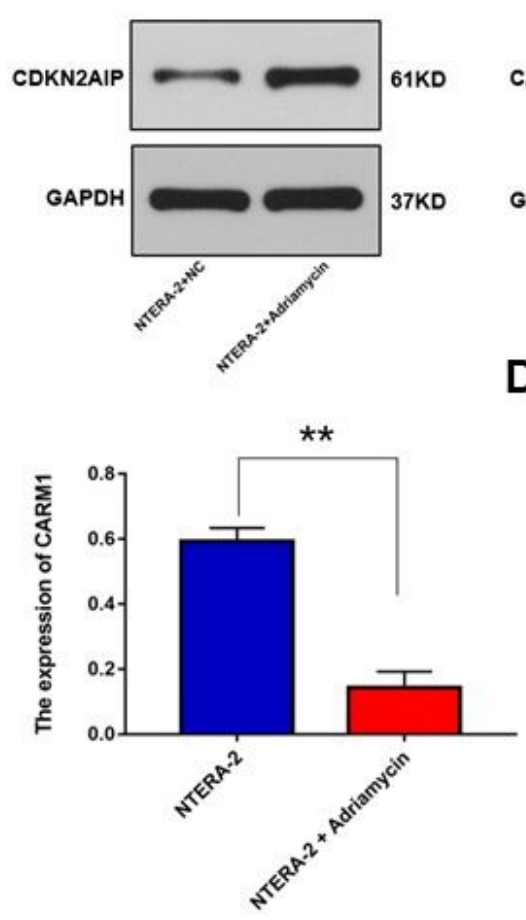

$\mathbf{F}$

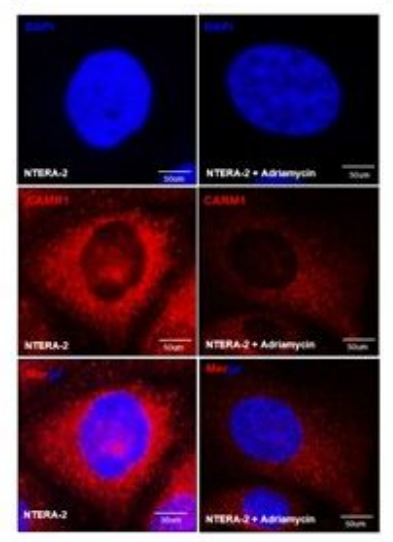

G

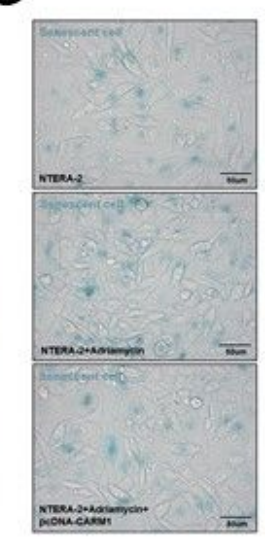

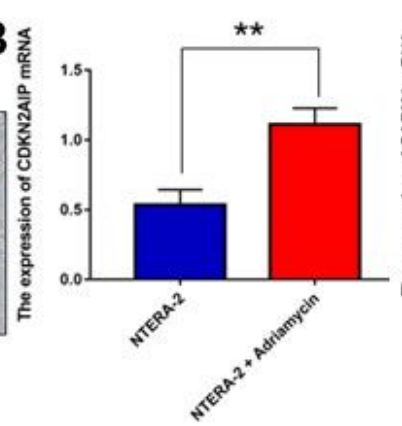
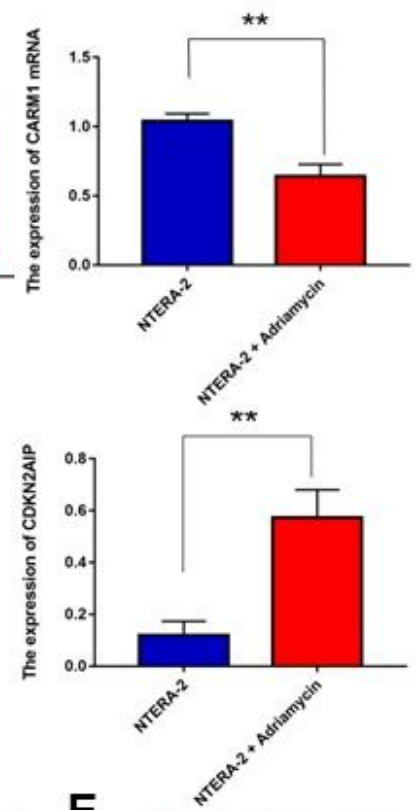

E

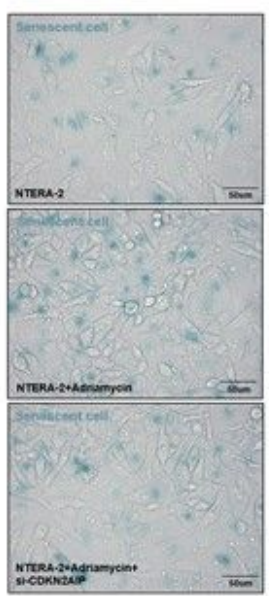

H

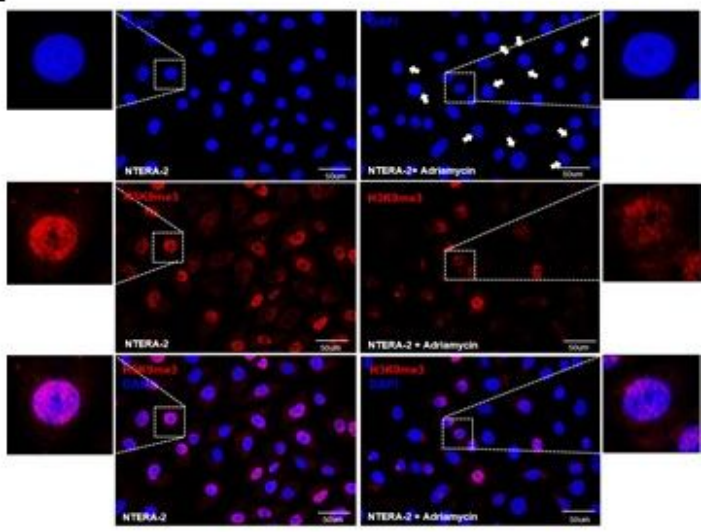

Figure 4 
Evaluation of cellular senescence by SA-beta-gal and H3K9me3 level detection assay on NTERA-2 cell line with modulated CDKN2AIP and CARM1 expression A. Senescence-associated beta-galactosidase (SA-beta-gal) activity assay on NTERA-2 cell line. Tumor cells were treated with sublethal concentrations of Adriamycin (ADM) to induce cellular senescence. B-C. qRT-PCR and western Blot analysis on CDKN2AIP and CARM1 protein expression in NTERA-2 cell line. Tumor cells were treated with sublethal concentrations of Adriamycin (ADM) to induce cellular senescence D. Immunofluorescence assay to detect CDKN2AIP in NTERA-2 cell line. Tumor cell groups were treated with or without sublethal concentrations of Adriamycin (ADM) to induce cellular senescence E. SA-beta-gal activity assay on NTERA-2 cell groups respectively treated with sublethal concentrations of Adriamycin (ADM), with or without simultaneous transfection of CDKN2AIP-specific siRNAs F. Immunofluorescence assay to detect CARM1 in NTERA-2 cell line. Tumor cell groups were treated with or without sublethal concentrations of Adriamycin (ADM) to induce cellular senescence G. SA-beta-gal activity assay on NTERA-2 cell groups respectively treated with sublethal concentrations of Adriamycin (ADM), with or without simultaneous transfection of CARM1 overexpression plasmid $\mathrm{H}$. Immunofluorescence assay to detect histone H3K9me3 level in NTERA-2 cell groups treated with or without sublethal concentrations of Adriamycin (ADM) to induce cellular senescence 

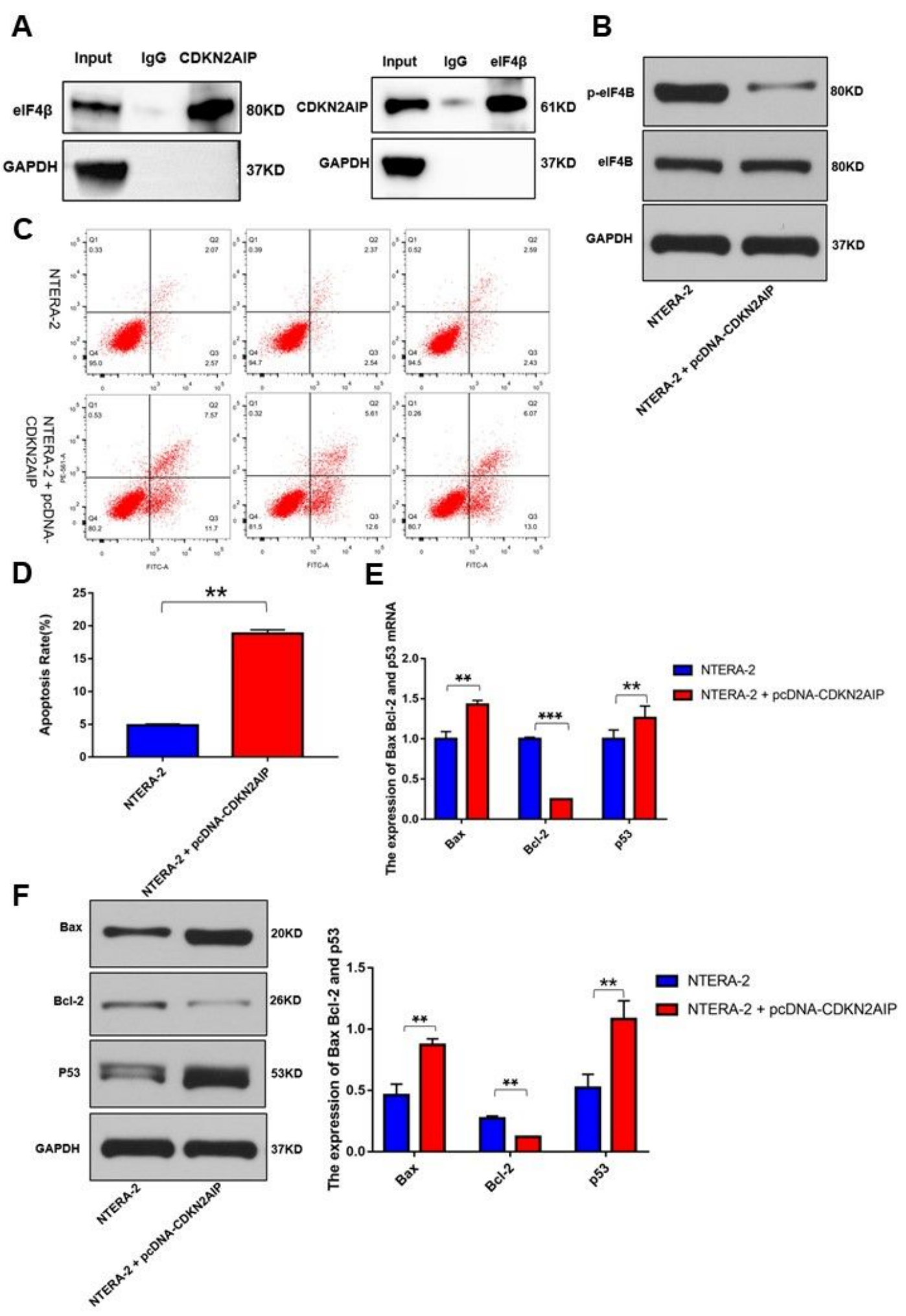

\section{Figure 5}

Co-IP detection of CKDN2AIP interaction with elF4B and cellular apoptosis analysis A. Co-IP assay to detect the interaction of CDKN2AIP protein with elF4B B. Western Blot analysis on elF4B and p-elF4B protein expression level in NTERA-2 cell groups respectively transfected with CDKN2AIP-specific overexpression plasmids C-D. Flow cytometric assay to evaluate NTERA-2 cellular apoptotic percentage, each cell group was respectively transfected with or without CDKN2AIP overexpression plasmids 
Immunofluorescent co-localization detection of CDKN2AIP and p53 in NTERA-2 cell line. Both CDKN2AIP and p53 plasmids were co-transfected into tumor cells E-F. mRNA and protein detections of several cellular apoptotic-related biological markers (Bax, BCL-2 and p53) in NTERA-2 cell groups respectively treated with or without CDKN2AIP specific overexpression plasmids or CKDN2AIP specific siRNAs

A

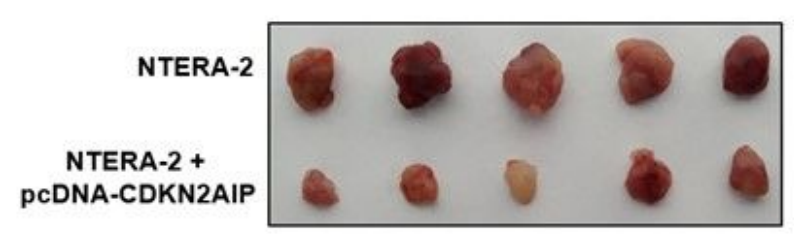

B

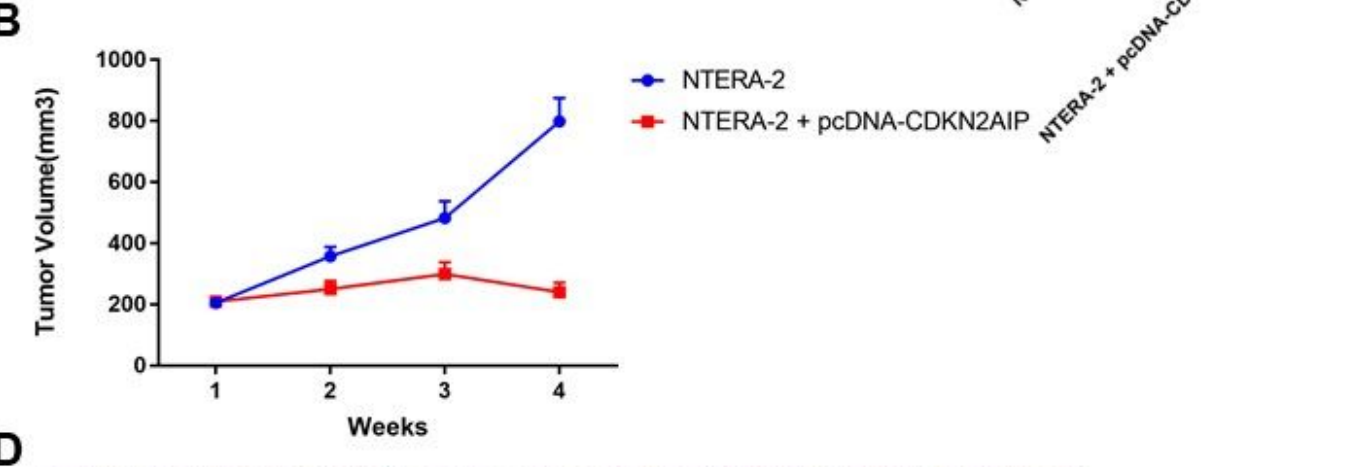

D

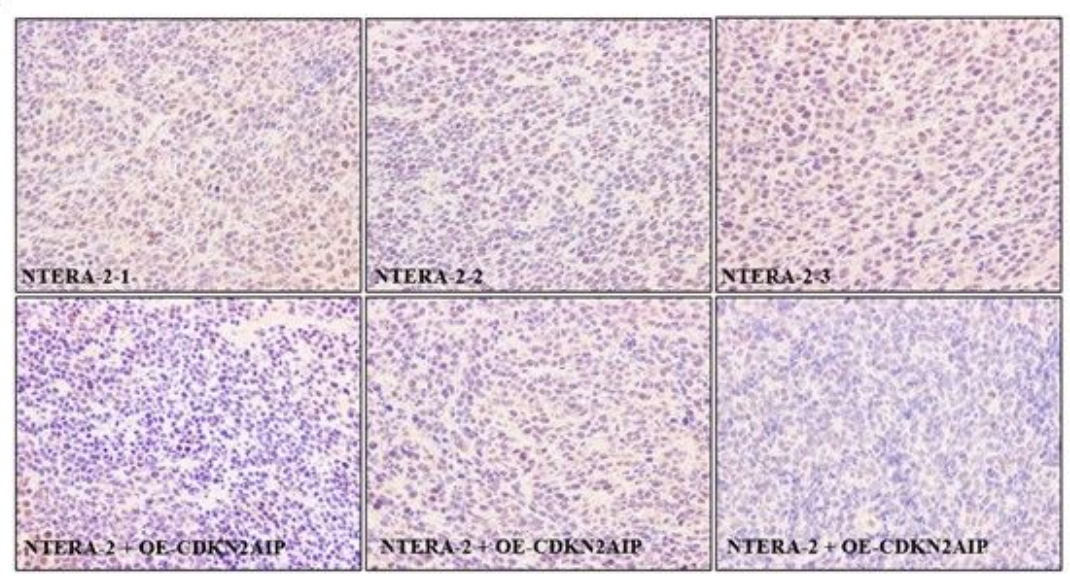

E

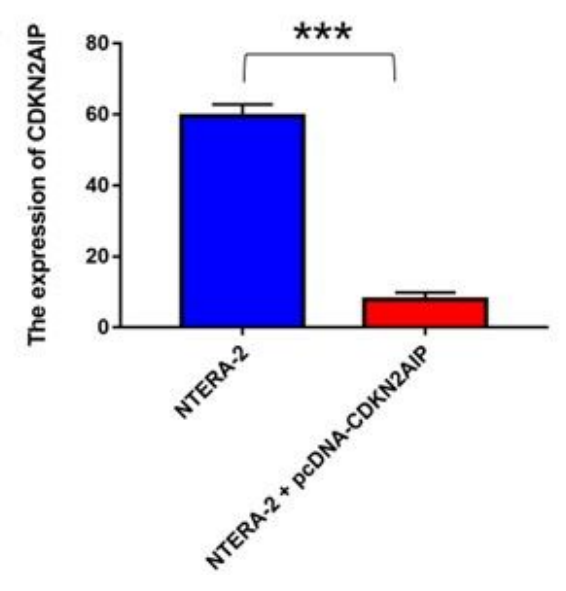

Figure 6 
NTERA-2 cell line SCID mice xenograft model experiment A. Xenograft model utilizing severe combined immune-deficient (SCID) mice. Each animal group was respectively inoculated with NTERA-2 tumor cells transfected with or without CDKN2AIP overexpression plasmids B-C. Tumor volume and weight change in xenograft SCID mice model. Each animal group respectively carried NTERA-2 tumor cells transfected with or without CDKN2AIP overexpression plasmids D-E. Immunohistochemical assay and quantitative analysis on CDKN2AIP expression level in xenograft tumor tissue of SCID mice xenograft models. Each animal group was respectively inoculated with NTERA-2 tumor cells transfected with or without CDKN2AIP overexpression plasmids

\section{Supplementary Files}

This is a list of supplementary files associated with this preprint. Click to download.

- GraphicalAbstract.jpg.jpg

- Rawdata.pdf

- Table1.docx

- Table2.docx

- Figures1.jpg

- FigureS2.jpg

- Figures3.jpg 